\title{
The Antioxidant Role of Glutathione and N-Acetyl- Cysteine Supplements and Exercise-Induced Oxidative Stress
}

\author{
Chad Kerksick $^{1}$ and Darryn Willoughby ${ }^{2}$ \\ ${ }^{1}$ Exercise and Sport Nutrition Laboratory and the ${ }^{2}$ Exercise and Biochemical Nutrition Laboratory, Baylor \\ University,Waco, TX. Address correspondence to Chad_Kerksick@baylor.edu
}

Received October 21, 2005/Accepted November 10, 2005

\begin{abstract}
An increase in exercise intensity is one of the many ways in which oxidative stress and free radical production has been shown to increase inside our cells. Effective regulation of the cellular balance between oxidation and antioxidation is important when considering cellular function and DNA integrity as well as the signal transduction of gene expression. Many pathological states, such as cancer, Parkinson's disease, and Alzheimer's disease have been shown to be related to the redox state of cells. In an attempt to minimize the onset of oxidative stress, supplementation with various known antioxidants has been suggested. Glutathione and $\mathrm{N}$-acetyl-cysteine (NAC) are antioxidants which are quite popular for their ability to minimize oxidative stress and the downstream negative effects thought to be associated with oxidative stress. Glutathione is largely known to minimize the lipid peroxidation of cellular membranes and other such targets that is known to occur with oxidative stress. N-acetyl-cysteine is a by-product of glutathione and is popular due to its cysteine residues and the role it has on glutathione maintenance and metabolism. The process of oxidative stress is a complicated, inter-twined series of events which quite possibly is related to many other cellular processes. Exercise enthusiasts and researchers have become interested in recent years to identify any means to help minimize the detrimental effects of oxidative stress that are commonly associated with intense and unaccustomed exercise. It is possible that a decrease in the amount of oxidative stress a cell is exposed to could increase health and performance. Journal of the International Society of Sports Nutrition. 2(2):38-44, 2005
\end{abstract}

Key Words: free radicals, reactive oxygen species, health, supplementation

\section{REACTIVE OXYGEN SPECIES AND OXIDATIVE STRESS}

The presence of oxygen is a fundamental component of cellular metabolism. However, any situation which results in a sudden or chronic overconsumption of oxygen can lead to the production of free radicals, which are more appropriately termed 'reactive oxygen species' (ROS). Production of these reactive oxygen species is known to occur as a result of a few different mechanisms: 1) mitochondrial origin in which free radicals either escape scavenging enzymes or develop due to an error in oxidative processes, 2) inside the capillary endothelium where a hypoxic and reoxygenation process is created during intense exercise as well as during various types of cardiovascular disease, and 3) an oxidative burst from inflammatory cells which are commonly mobilized as a result of the muscle or tissue damage which is well-documented with extended or eccentric-based exercise ${ }^{1}$ Aerobic energy metabolism, or oxidative phosphorylation, is a critical metabolic pathway within cells to provide the energy necessary to complete our daily tasks. In a resting state these processes work slowly while during times of intense exercise, these metabolic processes are increased as much as 100-fold. Inside the mitochondria, the electron transport is responsible for a series of redox reactions which result in the resynthesis of ATP. In this system, $\mathrm{O}_{2}$ is reduced by cytochrome-c oxidase, which is the terminal enzymatic component of this mitochondrial enzymatic complex. As the demand and subsequent flux for this process increases so does the chance that redox uncoupling will occur and increase the accumulation of free radicals throughout the cell. A free radical is a molecule that contains at least one unpaired electron in its outer spin orbits. Characterized by their unpaired electron(s), 
superoxide radicals, hydroxyl radicals, hydrogen peroxide, nitric oxide, lipid alkoxyl and peroxyl radicals are the most common reactive oxygen species in living, aerobic systems ${ }^{2}$. It is estimated that $2-5 \%$ of the oxygen that is passed through the electron transport system inside the mitochondria results in superoxide ${ }^{3}$. The complete reduction of oxygen can be seen from the steps outlined below ${ }^{4}$.

$$
\begin{array}{ll}
\mathrm{O}_{2}+\mathrm{e}^{-} \rightarrow \mathrm{O}_{2}^{-\bullet} & \text { Superoxide radical } \\
\mathrm{O}_{2}^{-\cdot}+\mathrm{H}_{2} \mathrm{O} \rightarrow \mathrm{HO}_{2}^{-}+\mathrm{OH}^{-} & \text {Hydroperoxyl radical } \\
\mathrm{HO}_{2}+\mathrm{e}^{-}+\mathrm{H} \rightarrow \mathrm{H}_{2} \mathrm{O}_{2} & \text { Hydrogen Peroxide } \\
\mathrm{H}_{2} \mathrm{O}_{2}+\mathrm{e}^{-} \rightarrow \cdot \cdot \mathrm{OH}+\mathrm{OH}^{-} & \text {Hydroxyl Radical }
\end{array}
$$

Superoxide is the most well-known of the free radicals as it is commonly produced during the natural pathway of oxidative phosphorylation. Superoxide is readily dismutated by intracellular superoxide dismutase enzymes (e.g. copper superoxide dismutase [CuSOD], magnesium superoxide dismutase [MgSOD]). Consequently, these antioxidant enzymes can have many different origins (e.g. endothelial, plasma, tissue) and are commonly used in the literature to assess the amount of oxidative stress that is occurring. Superoxide is converted primarily into hydrogen peroxide; however, from a chemical structure standpoint, hydrogen peroxide is not a free radical. It is considered to be a free radical due to its ability to readily result in the hydroxyl radical. Hydrogen peroxide, unlike other free radicals, is able to be transported across cellular membranes. The enzyme, catalase, has been shown to effectively dismantle much of the hydrogen peroxide found in our cells with water as a by-product. The hydroxyl radical is the most reactive of the free radicals and in the presence of various transition metals (e.g. $\mathrm{Fe}^{3+}, \mathrm{Cu}^{2+}$ ) it is known to directly target cellular lipids, proteins, nucleic bases, causing DNA base modification or fragmentation $^{5}$. The ability of the hydroxyl radical to remove or add hydrogen molecules to unsaturated aspects of cellular membranes (e.g. lipid peroxidation) makes it one of the most potent free radicals in existence. Its extremely short half-life restricts its diffusion capability to other parts of the cells while also enhancing its potency ${ }^{4}$.

To protect against the deleterious effects of ROS, our bodies have a complex system of endogenous antioxidant protection in the form of enzymes such as superoxide dismutase, catalase, and glutathione peroxidase. Under normal, resting conditions reactive oxygen species are removed from the cell preventing any subsequent damage. However, under more extreme conditions such as: 1) inadequate intake of foodstuffs containing the antioxidants, 2) excessive intake of pro-oxidants, 3) exposure to noxious chemicals or ultraviolet light, 4) injury/wounds, and/or 5) intense exercise, especially eccentric exercise, the body's endogenous antioxidant system is not able to effectively remove excessive ROS production ${ }^{2}$. In situations such as the ones listed above in which the production of prooxidant molecules increase to a point where the antioxidant system cannot effectively remove them is when oxidative stress is known to occur. Oxidative stress has been implicated in a number of diseases which include atherosclerosis, pulmonary fibrosis, cancer, Parkinson's disease, multiple sclerosis, and aging. $^{6}$. Research on oxidative stress during exercise has begun to indicate that regular training enhances the ability of these mechanisms to effective respond to the increase of oxidative product.

As mentioned before, free radicals have been found to react with macromolecules (lipids, proteins, DNA) within the cell, with one of the most frequent targets being the polyunsaturated fatty acids that largely comprise the cell membranes. The systematic oxidation of these polyunsaturated fatty acids is called lipid peroxidation. Lipid peroxidation has been found to limit different aspects of muscle or cell function by decreasing the fluidity of the membrane, making it more difficult for proteins/nutrients to pass through. Further, lipid peroxidation has been found to decrease the membrane's ability to uptake glucose as well as respond to varying levels of immune challenges ${ }^{7}$ Lipid peroxidation is commonly quantified in research studies by measuring the accumulation of the by-products that result from this process. One of these by-products is malondialdehyde (MDA). In response to various forms of exercise many studies have reported significant increases of malondialdehyde ${ }^{7-9}$. Evidence of lipid peroxidation by increased levels of malondialdehyde and other such substances such as 8-isoprostane and thiobarbarbituric acid-reactive substance levels is one of the primary means by which researchers have associated oxidative processes with an overall decrease of cellular function.

Supplementation with antioxidants, either through an increased consumption in the diet or from supplementation, has become extremely popular as a means to improve one's health or increase physical performance. It has been suggested that increasing the circulating levels of certain antioxidants (e.g. glutathione, n-acetyl-cysteine, $\alpha$-lipoid acid, vitamin A, vitamin E, vitamin C, etc.) will help to prevent the accumulation of free radicals inside our cells thus reducing oxidative stress $^{4,10,11}$ while other studies 
have suggested the possibly of little to no effect ${ }^{12}$. By decreasing oxidative stress, researchers have suggested that the risk of cancer, parkinson's disease, alzheimer's disease, etc. may all be decreased. Additionally, research has also begun to link excessive oxidative stress with an up-regulation of various proteolytic pathways (e.g. calcium-activated calpains and ubiquitin-proteolytic pathway) as well as apoptosis.

\section{GLUTATHIONE'S ROLE AS AN ANTIOXIDANT}

Glutathione is currently one of the most studied antioxidants. This is likely due to it being endogenously synthesized all throughout the body and it is basically found in all cells, sometimes in rather high concentrations. Investigations have highlighted many roles in which it is used including antioxidant defense, detoxification of electrophilic xenobiotics, modulation of redox regulated signal transduction, storage and transport of cysteine, regulation of cell proliferation, synthesis of deoxyribonucleotide synthesis, regulation of immune responses, and regulation of leukotriene and prostaglandin metabolism ${ }^{13}$. Many of these areas are highlighted below in the figure adopted from Sen and Packer to illustrate these roles of glutathione and to emphasize the importance of maintaining the redox state of the cell ${ }^{11}$.

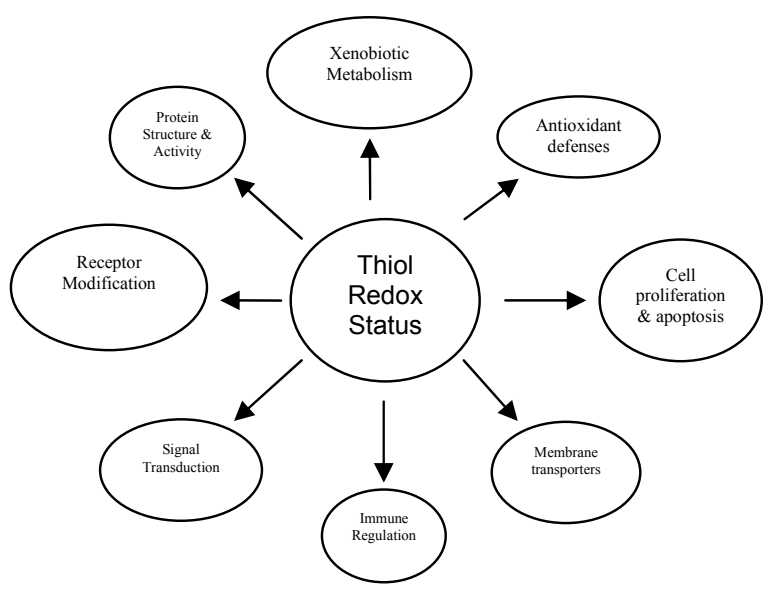

Figure 1. Thiol redox status and its many accompanying roles

\section{GLUTATHIONE RESPONSE TO EXERCISE}

Due to its large role in countering increases in lipid peroxidation which occurs during oxidative stress, especially during intense exercise, the response of glutathione has been frequently studied. Trained men who exercised to exhaustion on a treadmill had increased blood amounts of oxidized glutathione immediately after exercise, suggesting an increase in oxidative stress, but returned to rest after $1 \mathrm{~h}$. A decreased level of glutathione in the plasma is the result of an increased utilization of it inside the muscle, presumably to fight or "quench" any circulating free radicals. Other endurance-based exercise studies have reported similar results with Gohil et al. reporting an increase in oxidized glutathione after prolonged submaximal exercise with a concomitant decrease in reduced glutathione. Other studies have reported upon the changes seen in serum glutathione reductase, which is the enzyme responsible for restoring reduced glutathione from oxidized glutathione through the use of nicotinamide adenine dinucleotide (NADH) ${ }^{14,15}$. In these studies, an increase in glutathione reductase activity was thought to suggest an increase of oxidative stress induced by exercise. The potential for glutathione to minimize or reduce free radicals is apparent from the reported changes in glutathione status after exercise. In response to bouts of exercise, circulating levels of reduced glutathione are found to decrease while levels of oxidized glutathione increase. When considering the changes in redox chemistry occurring as a result of an increase in exercise, the ability of the body to maintain a proper balance of glutathione is quite important. Fortunately, in response to regular exercise training, resting levels of glutathione increase as an adaptation by the body to effectively deal with the increase in free radicals that commonly results secondary to the exercise training. While many of these studies utilized endurance-based exercise, much of the same response is expected when using resistance-based exercise. To date, few studies have been completed which utilized a typical resistance training program, but some studies using primarily eccentric contractions have been conducted. A study by Clarkson et al. had subjects complete 50 maximal contractions of the elbow flexors while having their blood taken prior to exercise, as well as immediately post-exercise and 24, 48, 72, 96 and $120 \mathrm{~h}$ post-exercise. They illustrated that increases in total glutathione in the plasma were found in those individuals who already had higher circulating levels, which they suggested was a greater adaptive response to the damaging exercise bout ${ }^{16}$. Another study which investigated the changes in glutathione status after exercise used $20-30$ y men who cycled for $40 \mathrm{~min}$ at $60 \%$ of their maximal oxygen consumption in an attempt to determine the role of glutathione as determinants of lipid peroxidation. Upon completion of the exercise bout oxidized glutathione levels increased by about $50 \%$ in response to the exercise bout suggesting a 
substantial onset of oxidative stress. Additionally reduced glutathione levels decreased by $13 \%$ after the exercise bout providing evidence that the intracellular glutathione was being utilized inside the muscle or associated tissues leading to a decrease in serum levels. The authors also concluded that resting lipid peroxidation levels were also able to predict the exercise induced changes in blood total glutathione in addition to resting oxidized glutathione levels being strongly related to their post-exercise values. The results from this study highlight the critical role that glutathione homeostasis has on modulating exercise induced oxidative stress, and conversely, the effect of oxidative stress at rest on exercise induced changes in glutathione redox status ${ }^{17}$.

Supplementation studies which have utilized exogenous forms of glutathione are not that common due to the poor ability of glutathione to stay intact throughout the cell with most of it being found to be degraded in the extracellular compartment ${ }^{18}$. Degradation of glutathione leads primarily to its constituent amino acids (e.g., cysteine, glycine), which can subsequently be used for glutathione synthesis inside the cell. One of the few studies that utilized exogenous glutathione used a combination of $1 \mathrm{~g}$ glutathione with $2 \mathrm{~g}$ vitamin $\mathrm{C}$ (another wellestablished antioxidant) daily for 7 days in trained athletes to test the possible effect on exercise-induced glutathione oxidation. As expected, progressive increases in exercise intensity resulted in an increase in oxidized glutathione of $34-320 \%$ compared to preexercise levels. Research involving glutathione suggests strongly that it has an important role in preventing oxidative stress-associated lipid peroxidation which subsequently makes maintaining an optimal balance of glutathione necessary for it to effectively quench peroxidation of the lipid membranes. Supplementation with glutathione has been met with little success as the bioavailability of glutathione is low due its transient transport throughout the cellular network. At the current time, the bioavailability of glutathione is thought to be extremely poor due to the hydrolytic enzymes that break down the glutathione upon ingestion. Nevertheless, popularity for glutathione administration and supplementation is high due to its primary role to minimize the oxidative stress seen with exercise.

\section{GLUTATHIONE AND SIGNAL TRANSDUCTION}

Another important role for glutathione relates to its impact over signal transduction of gene expression inside cells. Briefly, glutathione status has been favorably linked to two well-established redox sensitive transcription factors, nuclear factor $\mathrm{kB}(\mathrm{NK}-$ $\kappa \mathrm{B})$ and activator protein- $1^{19,20}$. Activation of NF- $\kappa \mathrm{B}$ appears from these studies to be critically regulated by intracellular thiol redox status. Extremely high or extremely low levels of oxidized glutathione results in less than optimal activation of these transcription factors making it important for optimal levels of intracellular oxidized glutathione to be maintained throughout the cell ${ }^{19,20}$. Tumor necrosis factor- $\alpha$ is a cytokine of various monocytes and macrophages and is also known to potently activate NF- $\mathrm{kB}$. Tumor necrosis factor- $\alpha$ is highly related to muscle wasting conditions such as cancer cachexia as well as AIDS and other muscle inflammatory conditions. Exhaustive exercise with athletes has been shown to increase levels of tumor necrosis factor- $\alpha$ in the serum creating a link between the muscle damage commonly seen with intense exercise and signal transduction of redox control parameters of the cell. Additionally, glutathione status has been investigated for its role in tumor necrosis factor- $\alpha$ induced activation of NF- $\kappa B$, which demonstrated that NF- $\kappa B$ activation is related to cellular glutathione levels ${ }^{21}$. Consequently, these studies provide continued support that maintaining optimal cellular levels of glutathione is important for effective cellular function.

\section{N-ACETYL-CYSTEINE}

$\mathrm{N}$-acetyl-cysteine (NAC) is an acetylated cysteine residue. An optimal thiol redox state has been demonstrated to be of primary importance if attempting to optimize the protective ability of the cell to oxidative stress. Relative to glutathione availability, one of the most important considerations has been to properly maintain the availability of cysteine in the blood as that is known to be the ratelimiting substrate for glutathione resynthesis ${ }^{3}$. Subsequently, identifying ways in which optimal availability of cysteine is achieved has been a primary approach in an effort to maintain the biosynthesis of reduced glutathione. Among the most widely used agents to maintain the cysteine pool is NAC in addition to $\alpha$-lipoic acid, which will not be discussed in detail. While other agents have been used, NAC and $\alpha$-lipoic acid are the most commonly utilized and discussed as a result of their proven safety and efficacy. In addition to the role glutathione and other thiols have on maintaining the cellular redox state, many studies have begun to explore if NAC supplementation can actually improve performance due to its ability to promote a more favorable cellular environment to achieve higher levels of performance. 
One of the first studies to utilize NAC to determine its role in improving muscle performance was conducted by Reid and colleagues. They pretreated subjects with n-acetyl-cysteine infusion $\left(150 \mathrm{mg} \cdot \mathrm{kg}^{-}\right.$ ${ }^{1}$ ) or a $5 \%$ dextrose placebo while undergoing an extended fatiguing bout of electrical stimulation of the ankle dorsiflexors. $\mathrm{N}$-acetyl-cysteine was found to have no impact over the nonfatigued muscle, but a significantly increased force output of approximately $15 \%$ was found after 3 minutes of repetitive contractions which persisted throughout the 30 minute protocol ${ }^{22}$. The authors concluded that NAC resulted in improved performance suggestive of oxidative stress having a causal role in the fatigue process. Another fatigue model using laboratory rats, NAC infusion $\left(150 \mathrm{mg} \cdot \mathrm{kg}^{-1}\right)$ and respiratory processes was conducted by Supinski et al. ${ }^{23}$ In this study they exposed the animals to an increased inspiratory load which was set up to induce fatigue of the respiratory muscle and ultimately end with respiratory arrest. $\mathrm{N}$-acetyl-cysteine infusion was found to better tolerate the respiratory loading by increasing the time to respiratory arrest. Additionally, diaphragm samples of both the nacetyl-cysteine and placebo groups demonstrated an attenuated decrease of reduced glutathione with those animals infused with NAC. This study provides evidence that NAC, a free radical scavenger, slows the rate of respiratory failure development during inspiratory resistive loading ${ }^{23}$. A recently published study out of Michael Reid's laboratory sought to determine the impact of NAC on fatiguing handgrip exercise. Participants were either infused with saline or NAC at a dosage of $150 \mathrm{mg} \cdot \mathrm{kg}-1$. While NAC was found to have no impact on force production during sustained maximal contractions, it was found to help reduce and inhibit glutathione oxidation during repetitive submaximal contractions. The authors concluded that NAC may be helpful at delaying fatigue as well as the building of oxidative stress, but more systemic, translational research on humans needs to be conducted. ${ }^{24}$ Furthermore, a study by Medved et al. used eight male subjects who were infused with either a placebo or NAC before and while cycling for 45 minutes at $71 \%$ peak $\mathrm{VO}_{2}$ and then to fatigue at $92 \%$ peak $\mathrm{VO}_{2}$ to determine any possible antioxidant or ergogenic effect ${ }^{25}$. Nacetyl-cysteine infusion resulted in a $26.3 \%$ increase in time to exhaustion at $92 \%$ peak $\mathrm{VO}^{2}$. Additionally, NAC infusion also resulted in increased total and reduced NAC levels in skeletal muscle at 45 minutes of exercise and at fatigue. The exercise protocol decreased the reduced glutathione levels with no impact on overall level of total glutathione ${ }^{25}$. Another study by the same authors used a similar infusion but this time with three higher intensity $45 \mathrm{~s}$ sprints with a fourth sprint at $130 \%$ peak oxygen consumption $^{26}$. While no change was illustrated in the time to fatigue at this higher level of intensity, NAC infusion did attenuate the reduction of reduced glutathione as well as the increase in oxidized glutathione suggesting that even with short bouts of high-intensity exercise, NAC is effective at promoting a positive redox balance within the cell ${ }^{26}$.

It has been well-reported that infusion of NAC can be effective at attenuating or minimizing muscle fatigue as well as enhance the overall redox status inside the cell. Several areas, however, still remain to be determined when it comes with NAC administration. The studies reported thus far have used exhaustion models of endurance-based exercise leaving the area of resistance exercise, specifically damaging eccentric exercise a major area of research to be conducted. It has been widely reported that an exercise bout consisting of eccentric muscle contractions can result in substantial damage to the contractile unit, which often results in inflammation, loss of $\mathrm{Ca}^{2+}$ homeostasis and possibly induction apoptosis as a result. A study by Childs et al. used eccentric contractions of elbow flexors to initiate a muscle damage response. Subjects were supplemented with either a placebo or a combination of the antioxidants vitamin $\mathrm{C}$ and NAC. In response to the muscle damage and supplementation, circulating levels of free iron (that could possibly react to form hydroxyl radicals) were increased, lactate dehydrogenase and creatine kinase, markers of protein breakdown and damage, were elevated in the supplemented group as well as an increase in markers for oxidative stress. Acute administration of the antioxidants vitamin $\mathrm{C}$ and NAC appear to facilitate the inflammatory and oxidative stress seen associated with muscle damage. It is possible that the amount of muscle damage and inflammation was so great during this study that administration of antioxidants had no possible benefit or as noted could possibly have had enhanced the response ${ }^{27}$. Currently, the relationship between muscle damage, oxidative stress and other systems of proteolysis such as apoptosis are poorly understood. A recent study by Quadrilatero and Hoffman-Goetz sought to determine the impact of NAC infusion on the development of apoptosis in rats that were infused with either saline or NAC and ran to exhaustion. The authors reported that the 90 minute exercise protocol increased intestinal lymphocytes phosphatidylserine concentration, mitochondrial membrane depolarization, and decreased intracellular glutathione concentration $(\mathrm{p}<0.05)^{28}$. Additionally, the rats that were infused with NAC better maintained their intracellular glutathione levels, prevented $(p<0.05)$ the 
externalization of phosphatidylserine, mitochondrial membrane depolarization and also prevented the loss of intestinal lymphocytes immediately and $24 \mathrm{~h}$ after exercise $^{28}$. A follow-up publication from the same study found that protein levels of caspase-3 and cytochrome c (pro-apoptotic markers) and bcl-2 (an anti-apoptotic marker) were significantly $(\mathrm{p}<0.05)$ elevated after exercise compared to the non-exercised controls $^{29}$. The authors concluded from this study that oxidative stress acting through a mitochondrial pathway may play a role in intestinal lymphocyte apoptosis after strenuous exercise.

$\mathrm{N}$-acetyl-cysteine is an effective scavenger of free radicals as well as a major contributor to maintenance of the cellular glutathione status in muscle cells. Studies have demonstrated some possible roles for NAC to minimize fatigue or extend the time it takes for it to accumulate as well as prevent the onset of apoptosis secondary to exhaustive exercise. It is still not known if the combination of vitamin $\mathrm{C}$ and NAC may be detrimental after damaging exercise as well as NAC's other possible roles during muscle damage and extensive muscle proteolysis.

\section{SUMMARY}

In conclusion, the development of free radicals and oxidative stress during exercise is an important consideration for optimal performance, recovery, and health. Currently, the relationship between oxidative stress and prolonged, unaccustomed, high-intensity exercise is not fully determined. Even further, research exists which illustrates a possible relationship between free radicals and oxidative stress to other diseases and pathways of cellular destruction. Systems of proteolysis and apoptosis are two of the primary pathways in which oxidative stress appears to play a substantial role in the extent to which they are active in skeletal muscle. A commonly sought-after approach to oxidative stress is the exogenous administration of compounds that are thought to have antioxidant properties. Much more research at this time needs to be conducted to determine the changes seen inside skeletal muscle cells after exposure to intense, unaccustomed damaging exercise. From these studies, researchers will be able to more effectively determine what signals or environments are responsible for causing oxidative stress, proteolysis as well as apoptosis. Additionally, future research should also target on the signal transduction pathways in skeletal muscle upon exposure to oxidative stress in an attempt to identify areas of cross-communication as possible areas for effective intervention.

\section{REFERENCES}

Evans WJ: Vitamin E, vitamin C, and exercise. Am J Clin Nutr 2000, 72(suppl):647S-652S.

Stipanuk MH: Biochemical and Physiological Aspects of Human Nutrition. Philadelphia, PA: SAUNDERS; 2000.

Sen CK: Antioxidant and redox regulation of cellular signaling: introduction. Med Sci Sports Exerc 2001, 33(3):368-370.

Clarkson PM, Thompson HS: Antioxidants: what role do they play in physical activity and health? Am J Clin Nutr 2000, 72(suppl):637S-646S.

5 Van Remmen H, Hamilton ML, Richardson A: Oxidative Damage to DNA and Aging. Exerc Sport Sci Rev 2003, 31(3):149-153.

6 Thannickal VJ, Fanburg BL: Reactive oxygen species in cell signaling. Am J Physiol Lung Cell Mol Physiol 2000, 279:L1005L1028.

7 Bryant RJ, Ryder J, Martino P, et al.: Effects of vitamin E and C supplementation either alone or in combination on exerciseinduced lipid peroxidation in trained cyclists. J Strength Cond Res 2003, 17(4):792-800.

8 Ramel A, Wagner KH, Elmadfa I: Plasma antioxidants and lipid oxidation after submaximal resistance exercise in men. Eur J Nutr 2004, 43(1):2-6.

9 Rodriguez MC, Rosenfeld J, Tarnopolsky MA: Plasma malondialdehyde increases transiently after ischemic forearm exercise. Med Sci Sports Exerc 2003, 35(11):1859-1865.

10 Chan KM, Decker EA: Endogenous skeletal muscle antioxidants. Critical Reviews in Food Science and Nutrition 1994, 34(4):403-426.

11 Sen CK, Packer L: Thiol homeostasis and supplements in physical exercise. Am J Clin Nutr 2000, 72(suppl):653S-669S.

12 Davison GW, Hughes CM, Bell RA: Exercise and mononuclear cell DNA damage: The effects of antioxidant supplementation. Int J Sport Nutr Exerc Metab 2005, 15:480-492.

13 Sen CK: Glutathione homeostasis in response to exercise training and nutritional supplements. Molecular and Cellular Biochemistry 1999, 196:31-42.

14 Viguie CA, Frei B, Shigenaga MK, et al.: Antioxidant status and indexes of oxidative stress during consecutive days of exercise. J Appl Physiol 1993, 75:566-572.

15 Laires MJ, Madeira F, Sergio J: Preliminary study of the relationship between plasma and erythrocyte magnesium variations and some circulating pro-oxidant and antioxidant indices in a standardized physical effort. Magnesium Research 1993, 6:233-238.

16 Lee J, Clarkson PM: Plasma creatine kinase activity and glutathione after eccentric exercise. Med Sci Sports Exerc 2003, 35(6):930-936. 
17 Laaksonen DE, Atalay M, Niskanen L, et al.: Blood glutathione homeostasis as a determinant of resting and exercise-induced oxidative stress in young men. Redox Rep 1999, 4(1-2):53-59.

18 Meister A: Glutathione deficiency produced by inhibition of its synthesis, and its reversal; applications in research and therapy. Pharmacol Ther 1991, 51:155-194.

19 Sen CK: Redox signaling and the emerging therapeutic potential of thiol antioxidants. Biochemical Pharmacology 1998, 55:17471758.

20 Sen CK, Packer L: Antioxidant and redox regulation of gene transcription. FASEB J 1996, 10(709-720)

21 Sen CK, Khanna S, Reznick AZ, et al.: Glutathione regulation of tumor necrosis factor-alpha-induced NF-kappa-B activation in skeletal muscle-derived L6 cells. Biochem Biophys Res Commun 1997, 237:645-649.

22 Reid MB, Stokic DS, Koch SM, et al.: N-Acetylcysteine inhibits muscle fatigue in humans. J Clin Invest 1994, 94:2468-2474.

23 Supinski GS, Stofan D, Ciufo R, et al.: N-acetylcysteine administration alters the response to inspiratory loading in oxygensupplemented rats. J Appl Physiol 1997, 82(4):1119-1125.

24 Matuszczak Y, Farid M, Jones J, et al.: Effect of n-acetylcysteine on glutathione oxidation and fatigue during handgrip exercise. Muscle Nerve 2005, 32:633-638.

25 Medved I, Brown MJ, Bjorksten AR, et al.: N-acetylcysteine enhances muscle cysteine and glutathione availability and attenuates fatigue during prolonged exercise in endurance-trained individuals. J Appl Physiol 2004, 97:1477-1485.

26 Medved I, Brown MJ, Bjorksten AR, et al.: N-acetylcysteine infusion alters blood redox status but not time to fatigue during intense exercise in humans. J Appl Physiol 2003, 94:1572-1582.

27 Childs A, Jacobs C, Kaminski T, et al.: Supplementation with vitamin C and N-Acetyl-Cysteine increases oxidative stress in humans after an acute muscle injury induced by eccentric exercise. Free Radical Biology \& Medicine 2001, 31(6):745-753.

28 Quadrilatero J, Hoffman-Goetz L: N-Acetyl-L-cysteine prevents exercise-induced intestinal lymphocyte apoptosis by maintaining intracellular glutathione levels and reducing mitochondrial membrane depolarization. Biochem Biophys Res Commun 2004, 319:894-901.

29 Quadrilatero J, Hoffman-Goetz L: N-Acetyl-L-Cysteine inhibits exercise-induced lymphocyte apoptotic protein alterations. Med Sci Sports Exerc 2005, 37(1):53-56. 